\title{
Studies on Aggregation of Molecular Chains and Gas Permeation for Poly(spiro[2,4]hepta-4,6-diene) Composed of Rigid and Semi-Rigid Sequences
}

\author{
Seiji HayAshi, Tisato KaJIYAma, and Motowo TaKayAnagi \\ Department of Applied Chemistry, Faculty of Engineering, Kyushu University \\ 36, Hakozaki, Higashi-ku, Fukuoka 812, Japan.
}

(Received August 12, 1980)

\begin{abstract}
Poly(spiro[2,4]hepta-4,6-diene) (PSHD) is an amorphous random copolymer composed of 1,2 and 1,4 units, whose sequences form semi-rigid (intermediate between rigid and flexible) and rigid chains, respectively. The density of PSHD decreases by increasing the fraction of 1,4 units $\left(F_{1,4}\right)$. The aggregation state of PSHD chains as a function of $F_{1,4}$ was investigated by Xray diffraction and electron microscopic observation. A heterogeneous amorphous state composed of densely and loosely packed aggregates changes to a more homogeneous one with increasing $F_{1,4}$. Permeabilities of $\mathrm{H}_{2}, \mathrm{He}, \mathrm{O}_{2}, \mathrm{Ar}$, and $\mathrm{N}_{2}$ gases through PSHD membranes with varying $F_{1,4}$ were measured. Permeability and diffusion coefficients for these gases exhibit minima in the vicinity of $F_{1,4}=0.7$. This fact can be explained by a combination of chain packing and heterogeneous aggregation of molecular chains.
\end{abstract}

KEY WORDS Poly(spiro[2,4]hepta-4,6-diene) / Rigid Polymer /

Amorphous Structure / Ion-Etching / Electron Microscopic Observation /

Permselective Membrane / Permeation of Gas / Diffusion of Gas /

Poly(spiro[2,4]hepta-4,6-diene) (PSHD) is an amorphous random copolymer composed of rigid sequences of 1,4 units and semi-rigid sequences of 1,2 units. Its chemical structure is,

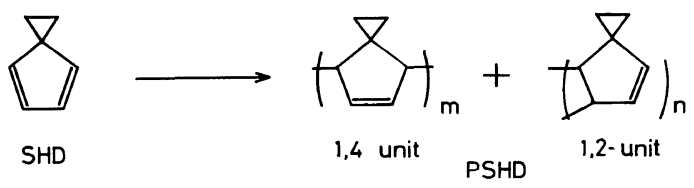

The characteristics of the 1,4 and 1,2 unit sequences were determined from viscoelastic measurement and rotational energy calculation. ${ }^{1}$ The term semi-rigid sequence means that the potential barrier between two minima on rotation about the $1,2-1,2$ bond is much lower than those for the 1,4-1,4 and 1,4-1,2 bonds and also that the 1,2-1,2 bond can be twisted only in a narrow range of rotational angle between these two minima. The density of PSHD is considerably lower than those of typical flexible polymers and decreases by increasing the fraction of 1,4 units, $F_{1,4}$, because the rigid sequences connected by the semi-rigid joints of the $1,2-1,2$ bonds cannot pack themselves densely and in parallel. Conformations that can be taken by the molecular chains are considerably restricted in comparison with those of completely flexible chains. ${ }^{1}$ If PSHD were composed all of 1,4 unit, its chains would align in parallel and crystallize without folding. Unfortunately, the synthesis of 1,4-PSHD has not as yet been successful for all the efforts under various polymerization conditions. However, it might be very interesting to compare the amorphous structure of PSHD composed of the rigid and semirigid sequences with those of flexible polymers, since the aggregation states of PSHD and ordinary flexible polymers must be quite different.

We suggest that it may be possible to prepare the PSHD film with characteristics of a permselective membrane for gases or liquids by controlling the inhomogeneity of the amorphous state. ${ }^{1}$ Permeation of gases through a polymeric membrane depends on the sorption and diffusion processes of gas molecules. The factors affecting it, i.e., solubility and diffusivity, have been correlated with the chemical structure and physical characteristics of the 
constituent molecules of the membranes. ${ }^{2}$ For instance, the factors, controlling physical properties, such as the glass transition behavior, ${ }^{3,4}$ molecular motions, ${ }^{5,6}$ degrees of crystallinity, ${ }^{7}$ crosslinking $^{8,9}$ and grafting ${ }^{10}$ affect the diffusivity of gases in the polymers. On the other hand, the factors controlling both chemical and physical properties such as plasticizer, ${ }^{11,12}$ and substituent groups ${ }^{13}$ affect also the solubility of gases into the polymeric membranes.

In this paper, the aggregation states of the PSHD molecules are investigated by X-ray diffraction and electron microscopic observation. We discuss the relation of the permeabilities and diffusivities of gases to the structural characteristics associated with rigid sequences jointed by semi-rigid sequences.

\section{EXPERIMENTAL}

Samples of PSHD were prepared according to the method established previously. ${ }^{1,14,15}$ PSHD membranes cast from a benzene solution were kept in vacuo more than one week.

NMR spectra were obtained with a Hitachi R24B high resolution NMR spectrometer in order to evaluate the fraction of 1,4 unit, $F_{1,4}$. X-ray diffraction diagrams of PSHD membranes were obtained with Ni-filtered $\mathrm{Cu} K \alpha$ radiation using a counter technique. Surfaces of the specimens were etched by oxygen and nitrogen ions for a given time with an Eiko Engineering ion-coater IB-2. A conventional platinum-carbon first stage replica of the ion-etched surfaces was used for observation by a Hitachi $\mathrm{H}$ 500 electron microscope.

Permeation properties of $\mathrm{H}_{2}, \mathrm{He}, \mathrm{O}_{2}, \mathrm{Ar}$, and $\mathrm{N}_{2}$ were investigated by a high vacuum method originally developed by Barrer ${ }^{16}$ in a temperature range from 298 to $338 \mathrm{~K}$. The permeation apparatus used is shown in Figure 1. Pressures on the upstream side were in the range of $10-60 \mathrm{cmHg}$. The increase in pressure on the downstream side was measured with a McLeod gage as a function of time. The diffusion coefficient of a given gas was evaluated by the timelag method. ${ }^{17}$

\section{RESULTS AND DISCUSSION}

\section{Aggregation States of PSHD Chains}

Figure 2 shows the $F_{1,4}$ dependence of the density

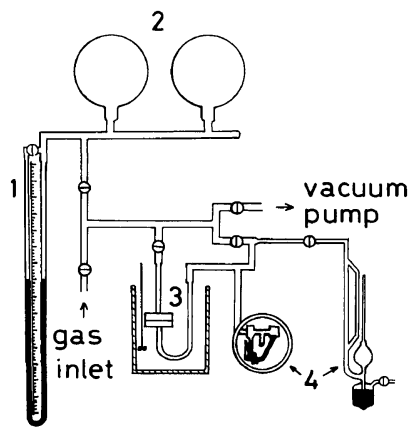

Figure 1. Permeation apparatus: 1, manometer; 2, reservoir of gas; 3 , permeation cell, 4; McLeod gage.

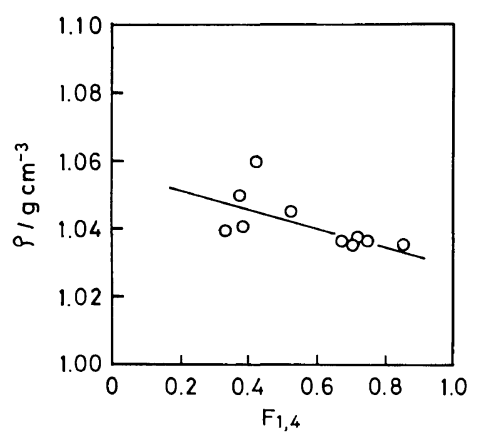

Figure 2. Density of PSHD measured at $303 \mathrm{~K}$.

of PSHD at $303 \mathrm{~K}$. The density decreases with increasing $F_{1,4}$. This means that the rigid 1,4sequences prevent close packing of the molecular chains, because the conformation of 1,2- and 1,4sequences is markedly limited. The density of PSHD is abnormally lower ${ }^{1}$ than that expected from a relation between density and the occupied volume of the repeating unit for typical polymers. ${ }^{18}$ It is expected from these results that the molecular packing or the aggregation state of PSHD greatly differs from those of flexible amorphous polymers.

Figure 3 shows X-ray diffractograms of PSHD membranes as a function of $F_{1,4}$. Intensity corrections for the background and the polarization factor were made by the ordinary method given in any standard textbooks. No special orientation of molecular chains was detected in the directions normal and parallel to the membrane surface, indicating completely random-chain orientation. A broad intensity distribution of amorphous halo having multiple peaks was observed for the samples with lower $F_{1,4}(=0.30$ and 0.43$)$. For the samples 


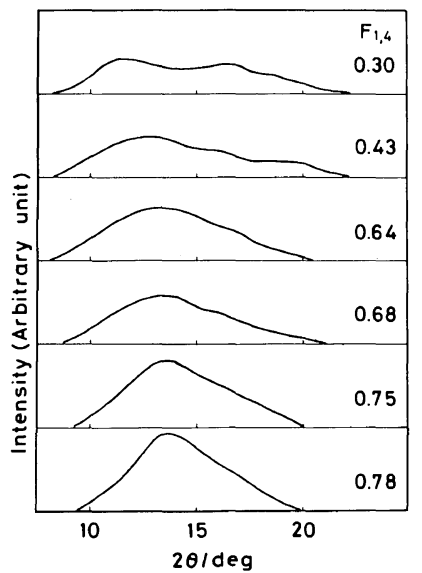

Figure 3. X-ray diffractograms of PSHD with various $F_{1,4}$.

with higher $F_{1,4}$, the amorphous halo was sharper. These facts indicate that the samples with lower fractions of 1,4 units have a broad distribution of mean intermolecular distances. It is apparent that the molecular aggregation state of PSHD is heterogeneous, that is, consisting of two regions in which the chains are densely and loosely packed. The aggregation state is more homogeneous for samples with higher $F_{1,4}$.

Figures 4 and 5 show electron micrographs of the surface replicas of the PSHD membranes with $F_{1,4}$ of 0.30 and 0.75 , respectively. The ion-etching technique $^{19}$ was employed to investigate the heterogeneity of molecular chain packing. The etching time was varied to clarify the effect of ion-etching. The surface of the specimen became clearer by increasing the degree of ion-etching. A remarkable difference in the morphology of the etched surface was observed between samples with higher and lower fractions of 1,4 units. Though a sample with $F_{1,4}=0.30$ initially had a relatively smooth surface, uneven surfaces composed of granular aggregates of diameter of about $20-40 \mathrm{~nm}$ became apparent with increasing etching time. These granular structures were similar to those reported by Frank et al. ${ }^{19}$ for polycarbonate specimens ion-etched after annealed below the glass-transition temperature. They pointed out that these grains are composed of regions characterized by a certain parallelism of chains. In the samples with low fractions of 1,4 units, however, the PSHD chains may not align in parallel but aggregate in a manner similar to random coils, since

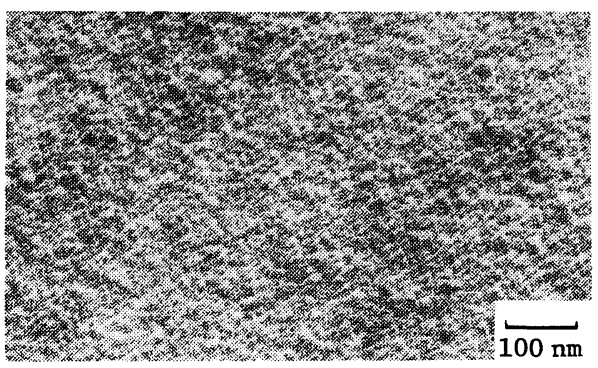

(a)

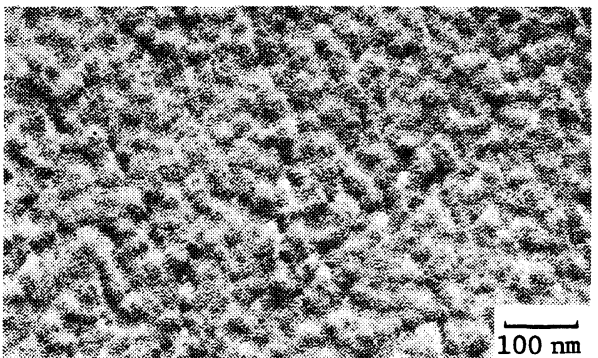

(b)

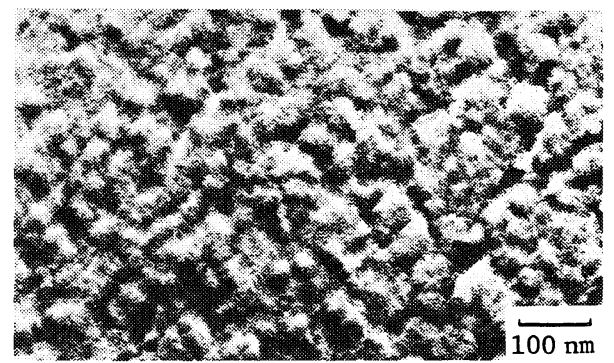

(c)

Figure 4. Electron micrographs of the surface of PSHD with $F_{1,4}=0.30$; ion-etched for (a) $0 \mathrm{~min}$, (b) $5 \mathrm{~min}$, and (c) $15 \mathrm{~min}$.

the bond angle of the 1,2 unit joining to 1,4 unit sequences is acute and this situation induces globular aggregates of chains during the chain-propagation reaction. It is reasonable to consider that loosely packed regions are less stable for ion-etching than densely packed ones, the former being destroyed by ionic collision even during a brief ion-etching treatment. Therefore, these granular structures must correspond to domains in which molecular chains are densely packed, but their conformations or intermolecular orders are not yet clear. The surface morphology of the ion-etched samples may indicate the degree of heterogeneity of molecular chain 


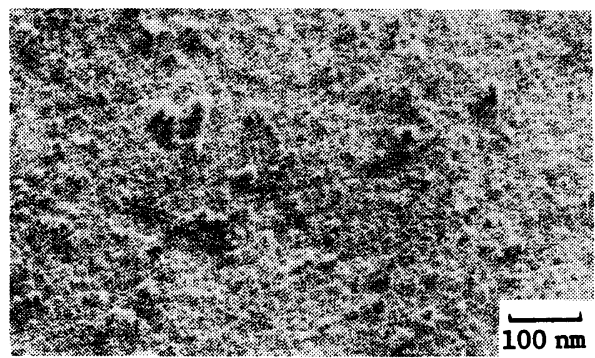

(a)

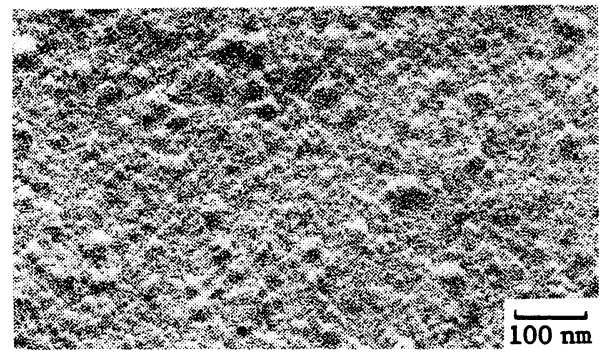

(b)

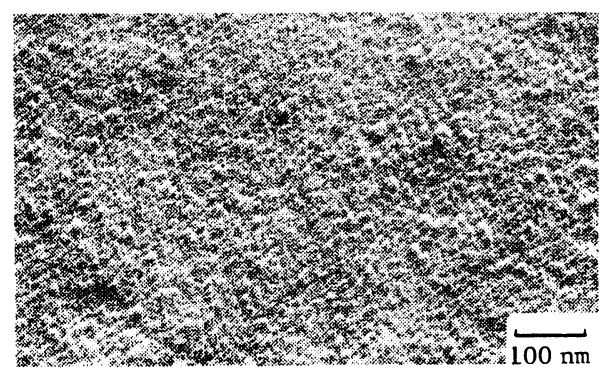

(c)

Figure 5. Electron micrographs of the surface of PSHD with $F_{1.4}=0.75$; ion-etched for (a) $0 \mathrm{~min}$, (b) $5 \mathrm{~min}$, and (c) $15 \mathrm{~min}$. packing, although it does not necessarily represent the internal structure. For the sample with $F_{1,4}$ of 0.75 , granules of about $10 \mathrm{~nm}$ in diameter were observed after etching for $15 \mathrm{~min}$, and the surface became gradually smoother as the ion-etching advanced. These observed morphologies of surface replica indicate that the heterogeneity with respect to molecular aggregation depends on the fraction of the rigid sequences. The state of chain aggregation in a PSHD sample with $F_{1,4}=0.30$ was more heterogeneous than in that of $F_{1,4}=0.75$. This agrees well with that from the $\mathrm{X}$-ray diffraction study shown in Figure 3.

The heterogeneity in the state of molecular aggregation was also confirmed by dynamic viscoelastic measurement as shown in Figure 6 of Reference 1. The $\alpha$ relaxation process, which is associated with the thermal motion of the sequential portion connected by 1,2 unit, was observed in $\tan \delta$ in a temperature range from 450 to $620 \mathrm{~K}$. The multiple $\alpha$ peaks in $\tan \delta$ were clearly observed for the samples with lower fractions of 1,4 units, and they gradually changed into a single peak by increasing the fraction of 1,4 units. The single relaxation process was observed for the samples with $F_{1,4}$ more than 0.52 in a temperature range from 530 to $550 \mathrm{~K}$. The appearance of the multiple $\alpha$ peaks is attributed to the heterogeneity of molecular aggregation.

Figure 6(a) and (b) show schematically the principal features of aggregation states of PSHD chains. Figure 6(c) shows a random-coil model of flexible chains. The bold and broken lines correspond to the rigid and semi-rigid sequences, respectively. Figure 6(a) shows the state of microscopically homogeneous aggregation (polymer chains with long

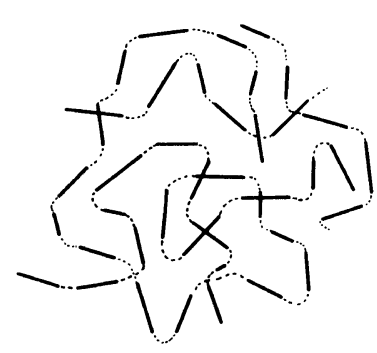

(a)

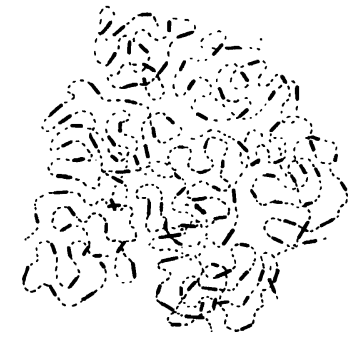

(b)

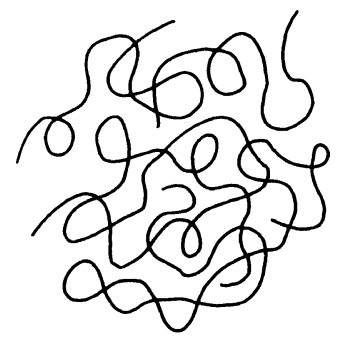

(c)

Figure 6. Models of molecular chain aggregation states for (a) polymer with long rigid sequences connected by semi-rigid sequences, (b) polymer with short rigid sequences connected by semi-rigid sequences (- - , rigid sequence; - - , semi-rigid sequence), and (c) flexible polymer (random coil). 
Permeation Property of Poly(spiro[2,4]hepta-4,6-diene)

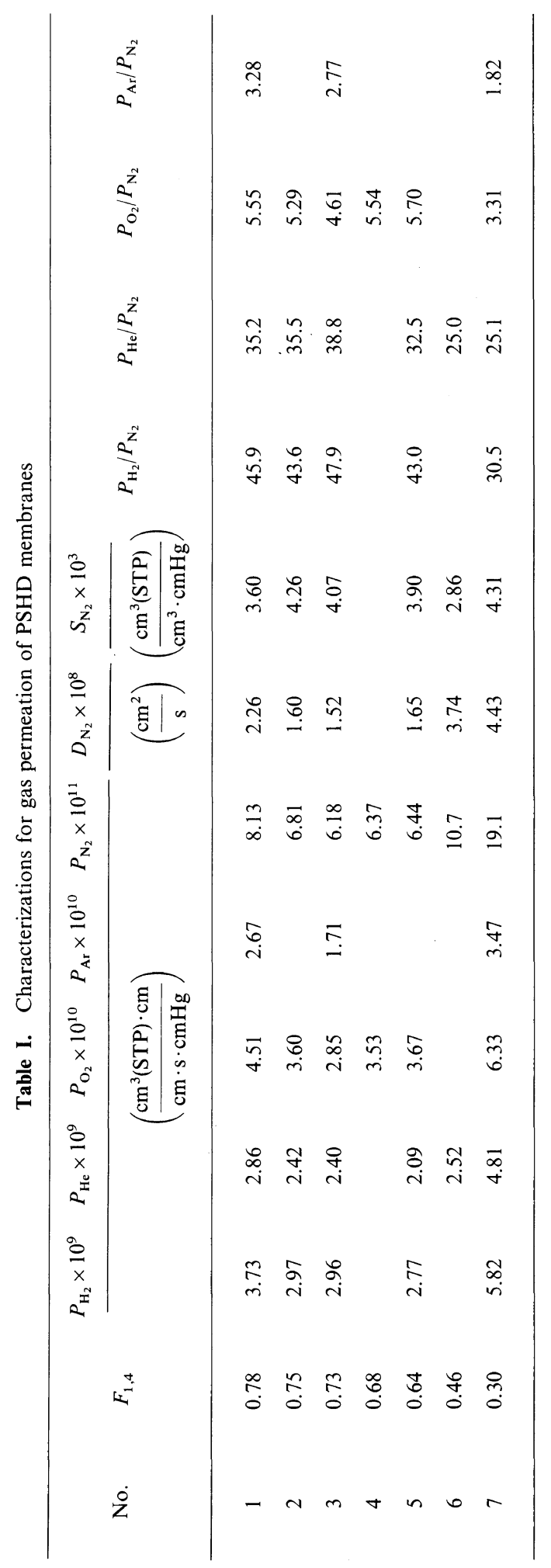


rigid sequences), while Figure 6(b) shows the state of microscopically heterogeneous aggregation (polymer chains with short rigid sequences). When long rigid sequences are connected by semirigid ones as shown in Figure 6(a), the free space increases due to the extremely restricted conformations of the chain. In the extreme case of $F_{1,4}=1.0$, the density would appreciably increase due to possible parallel alignment of the 1,4 unit sequences, which may form a liquid crystal. For those smaller $F_{1,4}$, the PSHD chains contract themselves and globular aggregation is more apparent as schematically shown by the change from Figure 6(a) to (b). The propagating ion-pair of the molecular chain may be confined in a globular molecular coil owing to the conformational restriction. This is suggested from the fact that the number-average molecular weight decreases with decreasing $F_{1,4} \cdot{ }^{1}$ The radius of gyration of the chains composed of 1,2 units may be fairly contracted due to the globular characteristics resulting from the acute bonding angle and the restricted rotational angles of the 1,2 units. Therefore, the chain expansion in the sample with $F_{1,4}=0.30$ is probably comparable with or less than that of unperturbed chains of flexible polymers. The morphological observation of Figure 4(c) apparently indicates that there exists microheterogeneity composed of the grain region of high density and the intergrain region of low density. Therefore, we propose the model of Figure 6(b) in which a globular molecular coil entangles itself to form a dense core and a loose shell interpenetrates the loose shells of the other molecules.

\section{Gas Permeability of PSHD}

The permeability of gases through PSHD membranes increases with an increase in temperature. This indicates that the PSHD membrane may be treated as a uniform membrane. We assume that Fick's law is obeyed in this system, because the interaction between the polymer and gas molecules studied here may be small. The relationship of $P=D \times S$ may be applied to PSHD membranes, where $P$ is the permeability coefficient, $D$, the diffusion coefficient, and $S$, the solubility coefficient. Of course, in order to clarify the permeation mechanism of gases in the PSHD membrane, $P, D$, and $S$ should be evaluated separately. We will leave the exact elucidation of this mechanism for future study.

Table I shows the permeability coefficients of $\mathrm{H}_{2}$, $\mathrm{He}, \mathrm{O}_{2}, \mathrm{Ar}$, and $\mathrm{N}_{2}$ gases, the diffusion coefficient and the solubility coefficient of $\mathrm{N}_{2}$ gas, and the ratio of the permeability coefficient of each gas to that of $\mathrm{N}_{2}$ gas $\left(P / P_{\mathrm{N}_{2}}\right)$ at $298 \mathrm{~K}$ as a function of $F_{1,4}$.

Figure 7 shows the $F_{1,4}$ dependence of the permeability coefficients for various gases at $298 \mathrm{~K}$.

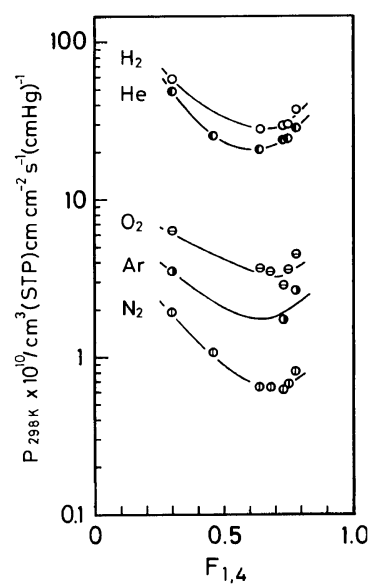

Figure 7. Semilogarithmic plots of permeability coefficients for $\mathrm{H}_{2}, \mathrm{He}, \mathrm{O}_{2}, \mathrm{Ar}$, and $\mathrm{N}_{2}$ gases vs. $F_{1,4}$ at $298 \mathrm{~K}$.

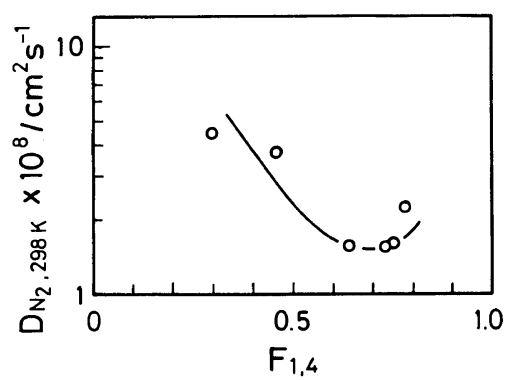

Figure 8. Semilogarithmic plot of diffusion coefficient for $\mathrm{N}_{2}$ gas vs. $F_{1,4}$ at $298 \mathrm{~K}$.

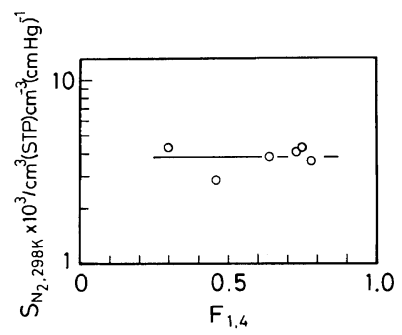

Figure 9. Semilogarithmic plot of solubility coefficient $(P / D)$ for $\mathrm{N}_{2}$ gas vs. $F_{1,4}$ at $298 \mathrm{~K}$. 
The characteristic trend is the appearance of a minimum in the vicinity of $F_{1,4}=0.7$. A similar trend was also observed for the diffusivity of $\mathrm{N}_{2}$ gas at $298 \mathrm{~K}$, as shown in Figure 8. Figure 9 shows a semilogarithmic plot of the solubility coefficient for $\mathrm{N}_{2}$ gas against $F_{1,4}$ at $298 \mathrm{~K}$. The solubility coefficients do not vary with $F_{1,4}$. The results imply that the $F_{1,4}$ dependence of the permeability coefficient mainly arises from that of the diffusion coefficient. Although the chain conformation-solubility relationship is not clear at the moment, the conformational difference of 1,2 unit and 1,4 unit must have direct effect on the diffusion process but not on the solution process.

The diffusion process is affected by the following factors: (1) the packing state of molecular chains and the heterogeneity of molecular aggregation and (2) the degree of thermal molecular motions. Because an increase in free space within the membrane enhances the gas diffusivity, a decrease in the density with increasing $F_{1,4}$ should give rise to a monotonic increase of $D$. However, the $F_{1,4}$ dependence of $D$ is the reverse of this expectation for the samples with smaller $F_{1,4}$ as shown in Figure 8 . Therefore, we must find another reason to explain the unexpected $F_{1,4}$ dependence of the gas diffusivity. This behavior cannot be explained by variation in thermal molecular motions, either. The $\tan \delta$ peak temperature for the $\alpha$ relaxation process of PSHD studied here is much higher than the measuring temperature $298 \mathrm{~K}$, at which the thermal motion is almost frozen. Therefore, the variation in thermal motion does not so much affect the diffusivity of gas as to explain the $F_{1,4}$ dependence of $D$. We consider the heterogeneity of molecular aggregation to elucidate this dependence. It is apparent that when two specimens with the same density are compared, the diffusivity in one with a more heterogeneous matrix is greater than that in the other with a less heterogeneous matrix, since gas molecules prefer to permeate through more loosely packed regions. The packing of PSHD molecular chains changes from heterogeneous to homogeneous with increasing $F_{1,4}$, as discussed above. Thus, the variations in density and heterogeneity with $F_{1,4}$ may lead to a minimum on the $F_{1,4}$ dependence of the diffusion or the permeability coefficient. As mentioned above, the diffusion of gas through the polymeric membrane sensitively reflects the internal structure of the chain packing or its heterogeneity as well as the thermal molecular motion of polymeric chains. Therefore, it is very worthwhile to examine the internal structure within polymeric membranes in terms of diffusion and/or permeation properties, in addition to $\mathrm{X}$-ray study or morphological observation.

Figure 10 shows semilogarithmic plots for the permeability coefficients for $\mathrm{H}_{2}, \mathrm{He}, \mathrm{Ar}$, and $\mathrm{O}_{2}$ gases relative to that of $\mathrm{N}_{2}$ gas against $F_{1,4}$ at $298 \mathrm{~K}$. Logarithms of $P_{\mathrm{H}_{2}} / P_{\mathrm{N}_{2}}, \quad P_{\mathrm{He}} / P_{\mathrm{N}_{2}}, \quad P_{\mathrm{Ar}} / P_{\mathrm{N}_{2}}$ and $P_{\mathrm{O}_{2}} / P_{\mathrm{N}_{2}}$ vary linearly with $F_{1,4}$. It is noteworthy that these ratios exhibit no maximum at the minimum point for $P$ in Figure 7 (corresponding to the arrows in Figure 10) nor decrease with increasing $P_{\mathrm{N}_{2}}$ in the range of higher $F_{1,4}$. It has been reported that the ratios of $P$ for several gases to $P_{\mathrm{N}_{2}}$ decrease with increasing $P_{\mathrm{N}_{2}}$ for membranes of butadieneacrylonitrile copolymer ${ }^{20}$ and for membranes of silicone and polycarbonate blend. ${ }^{21}$ Such a trend is generally recognized. However, for the PSHD membranes with high $F_{1,4}$, both permeabilities and permselectivities of gases increase with increasing $F_{1,4}$.

Figure 11 shows plots of $P / P_{\mathrm{N}_{2}}$ against $P_{\mathrm{N}_{2}}$ at $298 \mathrm{~K}$ for various polymers quoted from Polymer Handbook. $^{22}$ The data for the polymers in the rubbery state exhibit an inverse proportionality relationship on the double logarithmic plots, as

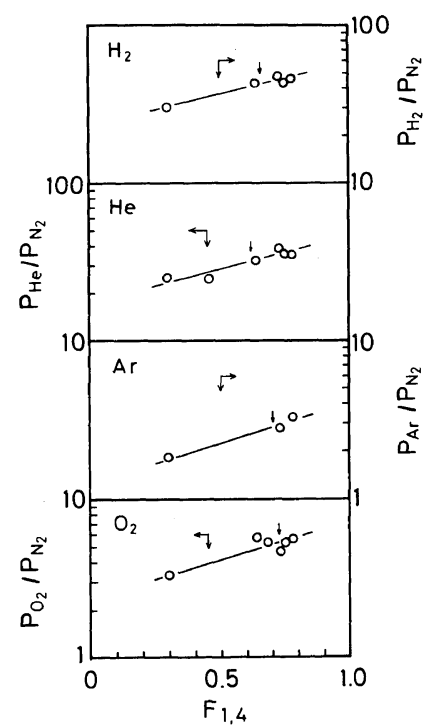

Figure 10. Semilogarithmic plots of ratios of permeability coefficients for various gases to nitrogen gas $v s$. $F_{1,4}$ at $298 \mathrm{~K}$. 


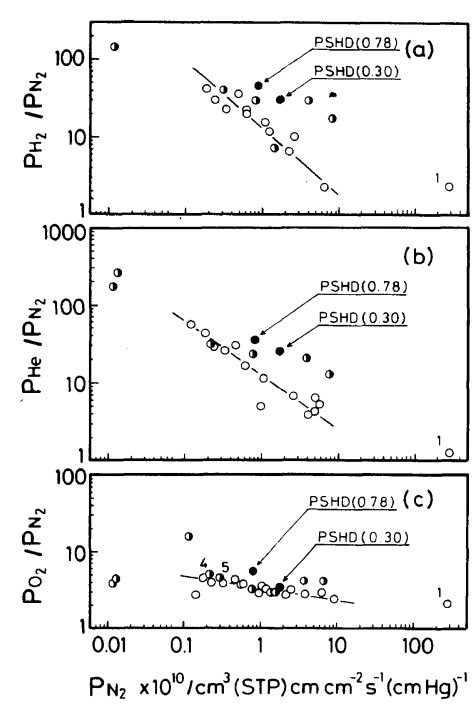

Figure 11. Plots of the ratio of permeability coefficients of (a) hydrogen, (b) helium, and (c) oxygen to nitrogen against permeability coefficient of nitrogen at $298 \mathrm{~K}$ : (○), rubbery polymers; ( $)$, glassy polymers; (1), poly(oxydimethylsilylene) with $10 \mathrm{wt} \%$ filler, vulcanized.

shown by unfilled circles, except for vulcanized poly(oxydimethylsilylene) containing $10 \mathrm{wt} \%$ filler. On the other hand, the data for the polymers in the glassy state are scattered as shown by half-filled circles. It is often found that the activation energy for permeation or diffusion changes discontinuously in the temperature range of glass transition..$^{2-4}$ This implies that the mechanism of diffusion or permeation distinctly changes with variation in thermal molecular motion. These two types of experimental results indicate that there is a distinct difference in the permeation mechanism of gases between glassy and rubbery polymers. The inverse proportionality of permselectivity $v s$. permeability shown in Figure 11 indicates that the remarkably large permeability and permselectivity cannot be expected for homogeneous polymeric membranes in the rubbery state. Since the permselectivity of glassy polymers deviates from this relation, superior permeability and permselectivity characteristics should be obtained with polymeric membranes in the glassy state rather than in the rubbery state. The ratios of the permeability coefficients of $\mathrm{H}_{2}, \mathrm{He}$, and $\mathrm{O}_{2}$ to $\mathrm{N}_{2}$ gases for the PSHD membranes of $F_{1,4}=0.30$ and 0.78 are also plotted in Figure 11. Since these ratios are considerably higher than those of other polymeric membranes except for $P_{\mathrm{O}_{2}} / P_{\mathrm{N}_{2}}$ for $F_{1,4}=0.30$, a glassy polymer containing rigid sequences may be utilized as a permselective membrane with excellent heat resistance.

\section{CONCLUSION}

From our study by X-ray diffraction, electron microscopic observations and dynamic viscoelastic measurement, we conclude that the aggregation state of PSHD with a low fraction of 1,4 units is very heterogeneous, being composed of low and high density regions. The degree of heterogeneity and the density decrease with increasing the fraction of 1,4 units. The permeability coefficients for $\mathrm{H}_{2}$, $\mathrm{He}, \mathrm{O}_{2}, \mathrm{~N}_{2}$, and Ar gases have minimum values in the vicinity of $F_{1,4}=0.7$. The diffusion coefficient for $\mathrm{N}_{2}$ gas has a similar tendency. The solubility coefficient, on the other hand, remains almost constant throughout the entire range of $F_{1,4}$. We conclude that the permeation of gas through PSHD membranes is primarily controlled by the $F_{1,4}$ dependence of the diffusion coefficient. Appearance of minima in the permeability coefficients at around $F_{1,4}=0.7$ may be explained by considering the following two factors: the density and the heterogeneity in the aggregation states. The increase in gas permeability with decreasing $F_{1,4}$ below 0.7 is due mainly to the fact that the aggregation state of PSHD molecular chains becomes more heterogeneous. In other words, it is associated with the advancement of phase separation between the loosely and densely packed regions. The increase in gas permeability by increasing $F_{1,4}$ above 0.7 is attributed mainly to the decrease in the density of PSHD. The permeability coefficients of $\mathrm{H}_{2}, \mathrm{He}$, and $\mathrm{O}_{2}$ relative to that of $\mathrm{N}_{2}$ gases for PSHD membrane are considerably high in comparison with those for other polymeric membranes.

\section{REFERENCES}

1. S. Hayashi, S. Ikuma, T. Kajiyama, and $M$. Takayanagi, J. Polym. Sci., Polym. Phys. Ed., 17, 1995 (1979).

2. J. Crank and G. S. Park, Ed., "Diffusion in Polymers," Academic Press, New York, N.Y., 1968.

3. P. Meares, J. Am. Chem. Soc., 76, 3415 (1954).

4. A. S. Michaels, W. R. Vieth, and J. A. Barrie, J. Appl. Phys., 34, 13 (1963).

5. T. Kajiyama, Y. Nagata, E. Maemura, and $M$. 
Takayanagi, Chem. Lett., 679 (1979).

6. T. Kajiyama, Membrane, 4, 229 (1979).

7. A. S. Michaels and R. B. Parker, Jr., J. Polym. Sci., 41, 53 (1959).

8. R. M. Barrer and G. Skirrow, J. Polym. Sci., 3, 549 (1948).

9. A. Aitken and R. M. Barrer, Trans. Faraday Soc., 51, 116 (1955).

10. R. Y. M. Huang and P. J. F. Kanitz, J. Appl. Polym. Sci., 13, 669 (1969).

11. C. A. Kumins, C. J. Rolle, and J. Roteman, J. Phys. Chem., 61, 1290 (1957).

12. P. R. Seibel and F. P. McCandless, Ind. Eng. Chem. Process. Dec. Dev., 11, No. 4, 470 (1972).

13. E. C. Marthin, P. D. May, and W. A. McMahn, $J$. Biomed. Mater. Res., 5, 53 (1971).

14. O. Ohara, C. Aso, and T. Kunitake, J. Polym. Sci., Polym. Chem. Ed., 11, 1917 (1973).
15. T. Kunitake, T. Ochiai, and O. Ohara, J. Polym. Sci., Polym. Chem. Ed., 13, 2581 (1975).

16. R. M. Barrer and G. Skirrow, J. Polym. Sci., 3, 549 (1948).

17. R. M. Barrer, Trans. Faraday Soc., 35, 628 (1939). (1939).

18. G. L. Slonimskii, A. A. Askadskii, and A. I. Kitaigorodskii, Polym. Sci., USSR, 12, 556 (1970).

19. W. Frank, H. Goddar, and H. A. Stuart, J. Polym. Sci., B., 5, 711 (1967).

20. G. J. Van Amerongen, J. Polym. Sci., 5, 307 (1950).

21. W. J. Ward III, W. R. Browall, and R. M. Salemme, J. Membr. Sci., 1, 99 (1976).

22. H. Yasuda and V. Stannett, "Polymer Handbook," second ed, J. Brandrup, and E. H. Immergut, Ed., John Wiley \& Sons, Inc., New York, N.Y., 1975, III229. 